\title{
Persistence of respiratory symptoms among COVID-19 survivors
}

\author{
Mohammed Mirazur Rahman, AKM Mosharraf Hossain, Mohammed Atiqur Rahman, \\ Shamim Ahmed, Rajashish Chakrabortty, Samprity Islam
}

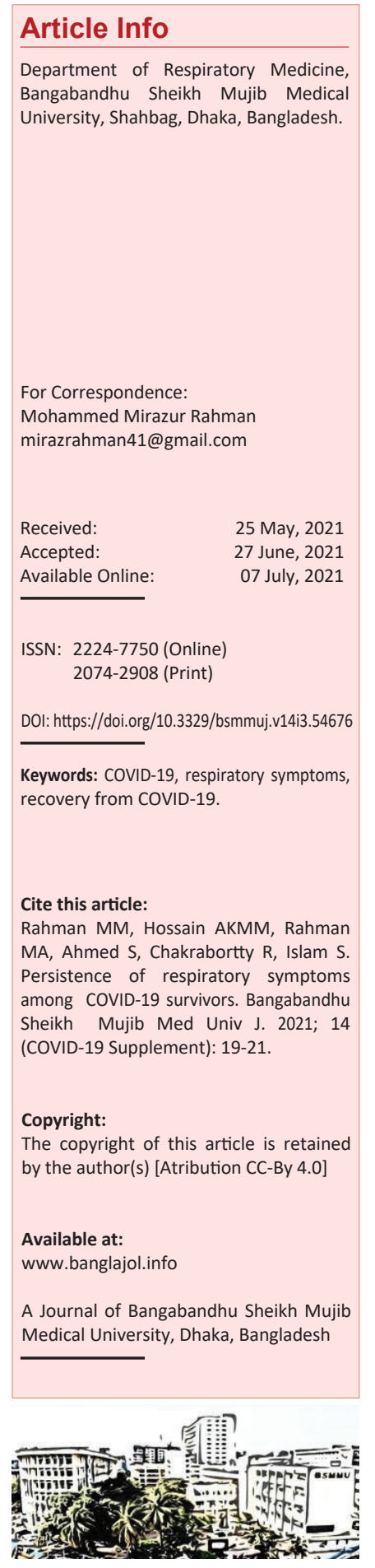

\section{Abstract}

This cross-sectional observational study was conducted among COVID-19 patients at Department of Respiratory Medicine, Bangabandhu Sheikh Mujib Medical University from July 2020 to December 2020. During follow-up around three months (90.62 \pm 2.21 days) after the onset of the first COVID-19 symptoms, 500 patients were taken as the study sample. Our study aimed to assess the persistent symptoms in patients who were discharged from the hospital after recovery from COVID-19. This study has shown that persistent respiratory symptoms were common after COVID-19 and the majority of patients were symptom-free three months after COVID-19 onset.

\section{Introduction}

Coronavirus Disease 2019 (COVID-19) is a new and highly contagious respiratory disease caused by severe acute respiratory syndrome coronavirus 2 (SARS-CoV-2), which presented a risk of infection from human to human. ${ }^{1}$ At the end of 2019, a novel coronavirus was identified as the cause of a cluster of pneumonia cases in Wuhan, a city in the Hubei Province of China. It rapidly spread, resulting in an epidemic throughout China, followed by an increasing number of cases in other countries throughout the world. In February 2020, the World Health Organization designated the disease COVID-19, which stands for coronavirus disease 2019. In Bangladesh, the first three known cases were reported on 8th March 2020 by the country's epidemiology institute, IEDCR. The spectrum of symptomatic infection ranges from mild to critical; most infections are not severe. ${ }^{2}$ After SARS-CoV-2 infection, a major complication of those who survived to COVID-19 outbreak is the development of severe lung disease leading to persistent respiratory symptoms. ${ }^{3}$ Persistent impairment of pulmonary function after discharge ranging from months to even years has been reported in other coronavirus infections such as severe acute respiratory syndrome (SARS) and the Middle East respiratory syndrome (MERS). ${ }^{4,5}$ Recent evidence has suggested that lung is the most affected organ by COVID-19. ${ }^{6}$ The extent and severity of the long term respiratory complications of COVID-19 infection remain to be seen, but emerging data indicate that many patients experience persistent respiratory symptoms months after their initial illness. ${ }^{7}$ Persistent respiratory symptoms following COVID-19 may cause substantial population morbidity, and optimal management remains unclear. Prospective studies are under way to evaluate these complications further and to identify people at greatest risk.

\section{Materials and Methods}

This was a Cross-Sectional observational study of the patients, who were recovered from acute COVID-19. Follow-up was conducted at the Department of Respiratory Medicine, Bangabandhu Sheikh Mujib Medical University, Dhaka July 2020 to December 2020. All patients admitted in the COVID-19 ward, who were laboratoryconfirmed SARS-CoV-2 infection by real-time reverse transcription polymerase chain reaction (RT-PCR) with respiratory symptoms were considered for this study. All patients who met the inclusion criteria (laboratoryconfirmed SARS-CoV-2 infection by RT-PCR with the presence of respiratory symptoms, 
age more than 18 years) and exclusion criteria (patients having history of pre-existing lung diseases like asthma, COPD, interstitial lung disease, bronchiectasis, obstructed sleep apnea, and history of pulmonary resection, neurological disease or mental illness) and World Health Organization criteria for discontinuation of quarantine were followed up. After discharge from the hospital, we kept their records. Patients were assessed around three months (90.62 \pm 2.21 days) after the onset of the first COVID-19 symptoms. In particular, data on specific respiratory symptoms potentially correlate with COVID-19 were obtained using a standardized questionnaire administered at enrollment. Patients were asked or requested to attend the post-COVID clinic three months from the onset of their first symptoms. Then data was collected with a structured questionnaire. The visual analog scale (VAS) was used to ask patients to score their quality of life from 0 (no symptoms) to 100 (worst symptoms imaginable) before COVID-19 and at the time of the visit. Total 590 confirmed COVID-19 patients were primarily screened for the study. During follow-up around three months after the onset of the first COVID-19 symptoms, 33 died and 57 patients were unwilling to participate. Following then 500 patients were taken as the study sample. After collecting the data it was checked and re-checked for omission, inconsistencies and improbabilities. Means and standard deviations (SD) were used to summarize continuous variables, while percentages were used for categorical variables. Data analysis was performed by Statistical Package for Social Science (SPSS), version-25.

\section{Results}

During follow-up around three months (90.62 \pm 2.21 days) after the onset of the first COVID-19 symptoms, among 500 patients the mean age of the participants was $52.69 \pm 12.81$ years, range $18-80$ years. Maximum patients132 (26.4\%) were over 60 years of age. Only $50(10.0 \%)$ patients were under 30 years and the maximum number of patients $334(66.80 \%)$ were male and the rest $166(33.20 \%)$ patients were female. Figure-1 shows the median number of respiratory symptoms during the infection reduced significantly over time (90 days later). Cough and shortness of breath were the most prevalent respiratory symptoms during the infection and at follow-up (cough: $88.4 \%$ versus $26.8 \%$; shortness of breath: $58.6 \%$ versus $22.6 \%$ ) respectively. Other prevalent respiratory symptoms were during the infection and follow-up ( sore throat: $22.6 \%$ versus $1.8 \%$, sputum production: $11.4 \%$ versus $2.2 \%$, anosmia: $30.8 \%$ versus $2.0 \%$, chest pain: $15.4 \%$ versus $5.6 \%$, rhinitis: $31.20 \%$ versus $2.0 \%$ ), respectively. Table-I shows majority of COVID-19 patients $300(60.0 \%)$ were symptoms free during follow-up (three months later), and $40.0 \%$ had persistent respiratory symptoms. In Table-II multivariate logistic regression analysis revealed that severity of COVID-19 during infection was independently and significantly $(p<0.05)$ associated with the persistent respiratory symptoms, having OR $=1.72$ (95\% CI 1.442-1.558).

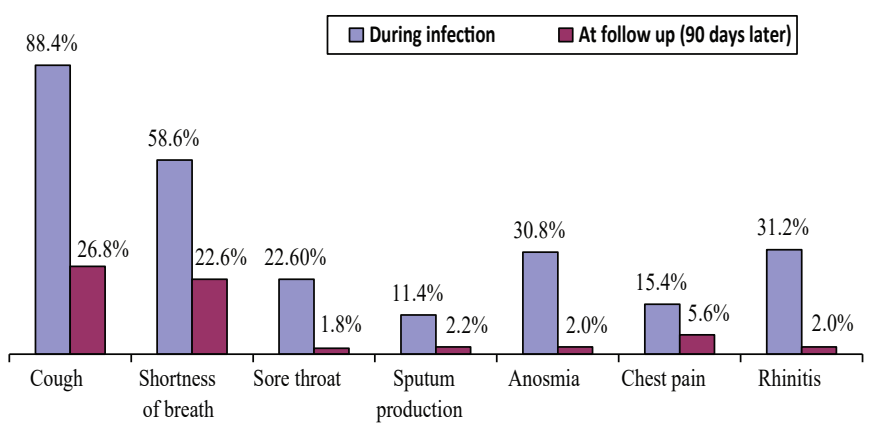

Figure-1: Bar graph showing the prevalence of respiratory symptoms during the infection and at follow-up (90 days later) of the study patients $(n=500)$

Table-I

Frequency of respiratory symptoms among COVID-19 survivors during follow-up (three months later)

\begin{tabular}{|l|c|c|}
\hline Respiratory symptoms & Number & Percentage \\
\hline Symptoms present & 200 & $40.0 \%$ \\
\hline Symptoms absent & 300 & $60.0 \%$ \\
\hline Total & 500 & $100.0 \%$ \\
\hline
\end{tabular}

\begin{tabular}{|l|c|c|c|}
\hline \multicolumn{4}{|c|}{ Table-II } \\
\hline \multicolumn{4}{|c|}{$\begin{array}{c}\text { Multivariate logistic regression analysis of risk factors } \\
\text { associated with the persistent respiratory symptoms }\end{array}$} \\
\hline Parameters & OR & 95\% CI & P value \\
\hline Age >60 years & 0.25 & $1.664-1.786$ & 0.64 \\
\hline Overweight & 0.029 & $1.466-1.604$ & 0.91 \\
\hline DM & 1.29 & $1.516-1.654$ & 0.39 \\
\hline Cardiovascular disease & 0.29 & $1.905-1.975$ & 0.56 \\
\hline Ground-glass opacity & 0.39 & $1.168-1.282$ & 0.75 \\
\hline Severity of COVID-19 & 1.72 & $1.422-1.558$ & $<0.001$ \\
\hline
\end{tabular}

\section{Discussion}

In the present study maximum patients were found in the sixth and seventh decades for both males and females. The mean age of the participants was $52.69 \pm 12.81$ years, range 
18-80 years. Among the study population, the maximum number of patients was male. Zhou, et al. conducted a cohort study of laboratory-confirmed admitted COVID-19 patients, their median age was 56.0 years and most patients were male. ${ }^{8}$ Our demographic findings, matched with this study. In the present study, we found the median number of respiratory symptoms during the infection reduced significantly over time (three months later). Cough and shortness of breath were the most prevalent respiratory symptoms during the infection and at follow-up. Other less frequent respiratory symptoms during the infection and follow-up were sore throat, sputum production, anosmia, chest pain, and rhinosinusitis. Goërtz, et al. conducted an observational study that showed fatigue and dyspnoea were the most prevalent symptoms during the infection and at follow-up. ${ }^{9}$ Other prevalent respiratory symptoms were cough, sore throat, anosmia, sneezing, and chest tightness. In our study, during follow-up majority was completely free of COVID-19 related respiratory symptoms. In another study conducted by Carfi, et al., patients have assessed a mean of 60.3 (SD, 13.6) days after onset of the first COVID-19 symptoms. ${ }^{3}$ All these results were quite similar to our result. In this study, we observed that there was no significant association of age, sex, co-morbidities and HRCT scan of chest with the persistent respiratory symptoms of COVID-19 survivors. But significant association was found between severity of COVID-19 and persistent respiratory symptoms. In multivariate logistic regression analysis also revealed that severity of COVID-19 during infection was independently and significantly associated with the persistent respiratory symptoms. These findings were similar to the observation done by Liu, et al. and Mowla, et al. ${ }^{10,11}$

\section{Conclusion}

This study has shown that persistent respiratory symptoms were common after COVID-19 and were related to age,sex, the severity of COVID-19, HRCT scan of chest and the number of co-morbidities present during the acute phase. The majority of patients were symptom-free three months after COVID-19 onset. Identification of persistent symptoms requiring early intervention is critical to plan for and providing post-acute medical, psychological, and physical services to enable recovery from COVID-19 infection, including the ability to return to work.

\section{Funding}

Support Self-funded

\section{Ethical Issue}

The protocol for this study was approved by the Institutional Review Board of Bangabandhu Sheikh Mujib Medical University

\section{Conflict of Interest}

Authors declare no conflict of interest

\section{References}

1. Chen Y, Liu Q, Guo D. Emerging coronaviruses: genome structure, replication, and pathogenesis. Journal of medical virology. 2020;92(4):418-23.

2. Huang C, Wang Y, Li X, Ren L, Zhao J, Hu Y, Zhang L, Fan G, Xu J, Gu X, Cheng Z. Clinical features of patients infected with 2019 novel coronavirus in Wuhan, China. The lancet. 2020; 395(10223):497-506.

3. Carfî A, Bernabei R, Landi F. Persistent symptoms in patients after acute COVID-19. Jama. 2020; 324(6): 603-5.

4. Xie L, Liu Y, Fan B, Xiao Y, Tian Q, Chen L, Zhao H, Chen W. Dynamic changes of serum SARS-coronavirus IgG, pulmonary function and radiography in patients recovering from SARS after hospital discharge. Respiratory research. 2005;6(1):1-7.

5. Hui DS, Wong KT, Ko FW, Tam LS, Chan DP, Woo J, Sung JJ. The 1-year impact of severe acute respiratory syndrome on pulmonary function, exercise capacity, and quality of life in a cohort of survivors. Chest. 2005;128(4):2247-61.

6. Shi H, Han X, Jiang N, Cao Y, Alwalid O, Gu J, Fan Y, Zheng C. Radiological findings from 81 patients with COVID-19 pneumonia in Wuhan, China: a descriptive study. The Lancet infectious diseases. 2020;20(4):425-34.

7. Haque MA. Pulmonary Fibrosis as Sequelae of Covid-19 Pneumonia. TAJ: Journal of Teachers Association. 2020; 33(2) :i-ii.

8. Zhou F, Yu T, Du R, Fan G, Liu Y, Liu Z, Xiang J, Wang Y, Song B, Gu X, Guan L. Clinical course and risk factors for mortality of adult inpatients with COVID-19 in Wuhan, China: a retrospective cohort study. The lancet. 2020;395 (10229):1054-62.

9. Goërtz YM, Van Herck M, Delbressine JM, Vaes AW, Meys R, Machado FV, Houben-Wilke S, Burtin C, Posthuma R, Franssen FM, van Loon N. Persistent symptoms 3 months after a SARS-CoV-2 infection: the post-COVID-19 syndrome?. ERJ open research. 2020;6(4).

10. Liu X, Yue X, Liu F, Wei L, Chu Y, Bao H, Dong Y, Cheng W, Yang L. Analysis of clinical features and early warning signs in patients with severe COVID-19: A retrospective cohort study. Plos one. 2020 Jun 26;15(6):e0235459.

11. Mowla SG, Azad KA, Kabir A, Biswas S, Islam MR, Banik GC, Khan MM, Rohan KI, Alam MA. Clinical profile of 100 confirmed COVID-19 patients admitted in dhaka medical college hospital, Dhaka, Bangladesh. Journal of Bangladesh College of Physicians and Surgeons. 20209: 29-36. 